\title{
BOUNDS ON VARIANCES OF LIFETIMES OF COHERENT AND MIXED SYSTEMS
}

\author{
KRZYSZTOF JASIŃSKI, ${ }^{*}$ Nicolaus Copernicus University \\ JORGE NAVARRO, ${ }^{* *}$ Universidad de Murcia \\ TOMASZ RYCHLIK, ${ }^{* * *}$ Nicolaus Copernicus University and Polish Academy of Sciences
}

\begin{abstract}
We consider coherent and mixed reliability systems composed of elements with independent and identically distributed lifetimes. We present upper bounds on variances of system lifetimes, expressed in terms of variances of single components. We also discuss attainability conditions and some special cases and examples.
\end{abstract}

Keywords: Coherent system; mixed system; variance; Samaniego signature; upper bound 2000 Mathematics Subject Classification: Primary 62N05; 60E15; 62G30

\section{Introduction}

Coherent systems provide useful mathematical models for sophisticated technical devices composed of simple elements. The whole device can operate as long as given selections of its elements work. It is assumed that the lifetime of the system is a function of the component lifetimes. Barlow and Proschan (1975) is the classic monograph devoted to this topic. A significant contribution to the analysis of the system lifetime was made by Samaniego (1985), who proved that if the component lifetimes are independent and identically continuously distributed then the distribution of the system lifetime is a mixture of the distributions of the so-called $k$-out-of- $n$ systems. The $k$-out-of- $n$ system, $k=1, \ldots, n$, is a system that functions as long as at least $k$ of its $n$ components function. The mixture coefficients merely depend on the structure function of the system. The distributions of $k$-out-of- $n$ systems depend on the parent distribution of the single component in a simple way. The Samaniego representation was further extended to systems with exchangeable component lifetimes (cf. Navarro and Rychlik (2007) and Navarro et al. (2008b)). The formula allows us to represent the distributions of lifetimes of reliability systems as fixed combinations of lifetime distributions of $k$-out-of- $n$ systems. Boland and Samaniego (2004) introduced the notion of mixed systems by admitting signatures with arbitrary nonnegative coordinates summing up to 1 . The mixed system is a randomly chosen $k$-out-of- $n$ system with the choice probability determined by the signature. The role of signatures in reliability theory was comprehensively reviewed in Samaniego (2007).

The lifetime of a $k$-out-of- $n$ system is the same as that of the $k$ th greatest order statistic of the component lifetimes. Accordingly, the problem of evaluating expectations of system lifetimes is

\footnotetext{
Received 3 March 2009; revision received 17 June 2009.

* Postal address: Faculty of Mathematics and Computer Science, Nicolaus Copernicus University, Chopina 12, 87100 Toruń, Poland. Email address: krzys@mat.uni.torun.pl

** Postal address: Facultad de Matematicas, Universidad de Murcia, 30100 Murcia, Spain.

Email address: jorgenav@um.es

*** Postal address: Institute of Mathematics, Polish Academy of Sciences, Chopina 12, 87100 Toruń, Poland.

Email address: trychlik@impan.gov.pl
} 
equivalent to evaluating expectations of linear combinations of order statistics. Various bounds on expected system lifetimes composed of elements with lifetime distributions coming from various nonparametric families were reviewed in Rychlik (2008a). Respective bounds for the systems with exchangeable components were presented in Navarro and Rychlik (2007).

Papadatos (1995) originally exploited the Hoeffding representation of covariance (see Hoeffding (1940) and Lehmann (1966, Lemma 2) for a short proof) in order to establish sharp bounds on variances of order statistics (and so the $k$-out-of- $n$ systems) of independent and identically distributed (i.i.d.) samples. They were further specified by Papadatos (1997) to the case of order statistics of symmetric populations. A similar approach was used in Klimczak and Rychlik (2004) for evaluating variances of $k$ th record values. Rychlik (2008b) determined sharp bounds on variances of order statistics coming from dependent, identically distributed populations. We also mention several generalizations of the Hoeffding lemma to covariances of functions of random variables (see Quesada-Molina (1992) and Cuadras (2002)), multivariate versions (see Block and Fang (1988), Yu (1993), Prakasa Rao (1998), and Beare (2009)), and higher-moment representations (see Mardia and Thompson (1972) and Jones and Balakrishnan (2002)).

In this paper we use the ideas of Papadatos (1995) to establish upper bounds on variances of lifetimes of coherent and mixed systems composed of elements with i.i.d. lifetimes. The bounds presented in Section 2 depend on the variance of a single-component lifetime, and are expressed in terms of the maximum of a polynomial of two variables, with coefficients depending only on the system signature, over a triangular domain. We further present a method of calculating the maximum, and present conditions of attainability of the bounds by coherent and mixed systems. In Section 3 we discuss the important special cases of systems with monotone and unimodal signatures for which our bounds are attained. We also formulate a conjecture that our variance bounds are sharp for all 'deterministic' coherent systems, and show an example of a mixed system for which our bound can be improved. We say that the system determined by a signature is 'deterministic' if it is possible to construct (and choose deterministically) one coherent system with the given signature. Otherwise, we call the system 'random'.

\section{General results}

We assume that a coherent system with a given structure function is composed of $n$ identical elements. The lifetimes of the components, $X_{1}, \ldots, X_{n}$, are independent and have a common continuous distribution function $F$ and positive finite variance $\sigma^{2}$, say. It is well known (see Samaniego (1985), (2007)) that the distribution function of the system lifetime $T$ has the form

$$
\mathrm{P}(T \leq x)=\sum_{i=1}^{n} s_{i} \mathrm{P}\left(X_{i: n} \leq x\right),
$$

where $X_{1: n} \leq \cdots \leq X_{n: n}$ are the order statistics of lifetimes $X_{1}, \ldots, X_{n}$, and

$$
s_{i}=\mathrm{P}\left(T=X_{i: n}\right), \quad i=1, \ldots, n .
$$

The vector $\boldsymbol{s}=\left(s_{1}, \ldots, s_{n}\right)$ is called the Samaniego signature of the system, and it merely depends on the structure function of the system, and is independent of the distribution of the component lifetimes. We have $s_{i} \geq 0, i=1, \ldots, n$, and $\sum_{i=1}^{n} s_{i}=1$. Clearly, the distributions of the order statistics depend on the marginal $F$, and do not depend on the structure of the system. These distributions are conveniently expressed by means of the Berstein 
polynomials,

$$
B_{k, n}(u)=\left(\begin{array}{l}
n \\
k
\end{array}\right) u^{k}(1-u)^{n-k}, \quad 0<u<1,0 \leq k \leq n,
$$

as follows:

$$
\mathrm{P}\left(X_{i: n} \leq x\right)=\sum_{k=i}^{n} B_{k, n}(F(x)), \quad i=1, \ldots, n .
$$

Each distribution function is the composition $G_{i: n}(F(x))$ of the distribution function $G_{i: n}$, say, the $i$ th order statistic of a standard uniform sample of size $n$, and the parent $F$. The former has density

$$
g_{i: n}(u)=n B_{i-1, n-1}(u), \quad 0<u<1 .
$$

Accordingly, (1) can be written as

$$
\mathrm{P}(T \leq x)=G(F(x))
$$

where

$$
G(u)=\sum_{i=1}^{n} s_{i} G_{i: n}(u)=\sum_{i=1}^{n} s_{i} \sum_{k=i}^{n} B_{k, n}(u)=\sum_{k=1}^{n}\left(\sum_{i=1}^{k} s_{i}\right) B_{k, n}(u) .
$$

Function (5) is a polynomial of degree $n$ that strictly increases on the interval $[0,1]$, and satisfies $G(0)=0$ and $G(1)=1$. It depends on the Samaniego signature of the system, which we drop in our notation for brevity. The function can be also written in the simpler form $G(u)=\sum_{i=1}^{n} b_{i} u^{i}$, useful in calculations. Note that the boundary conditions imply that $G$ does not have a constant term and that $\sum_{i=1}^{n} b_{i}=1$. The vector $\boldsymbol{b}=\left(b_{1}, \ldots, b_{n}\right)$ with possibly negative coordinates is called the maximal signature of a system. This notion was introduced and exploited in the context of analyzing reliability systems in Navarro et al. (2007). The derivative of (5),

$$
g(u)=\sum_{i=1}^{n} s_{i} g_{i: n}(u)=n \sum_{i=0}^{n-1} s_{i+1} B_{i, n-1}(u),
$$

is a positive polynomial of degree $n-1$. The function $p(u)=1-G(1-u)$ is called the domination polynomial of the system (cf., e.g. Navarro et al. (2008a)).

Important examples of coherent systems are the so-called $k$-out-of- $n$ systems, which function as long as at least $k$ of their $n$ components function. The lifetime of the $k$-out-of- $n$ system is $T=X_{n+1-k: n}$, and its signature consists of $s_{n+1-k}=1$ and $s_{j}=0, j \neq n+1-k$. By (1), the distribution of the system lifetime is the same as that of a randomly chosen $k$-out-of- $n$ system with probabilities $s_{n+1-k}, k=1, \ldots, n$. All the systems of sizes $n=3$ and 4 were presented in Kochar et al. (1999) and Shaked and Suarez-Llorens (2003), respectively. Navarro and Rubio (2009) proposed an algorithm for determining all the coherent systems of a fixed size, and presented the exhaustive set of 180 coherent systems of size $n=5$. The number of systems increases rapidly as the size increases. Navarro and Rubio (2009) proved that there are 16145 systems with six components.

Boland and Samaniego (2004) proposed the notion of mixed systems, which arise by randomly choosing a $k$-out-of- $n$ system with arbitrary probabilities $s_{n+1-k}, k=1, \ldots, n$, from the simplex $8^{n}=\left\{\left(s_{1}, \ldots, s_{n}\right): s_{i} \geq 0, \sum_{i=1}^{n} s_{i}=1\right\}$. Evidently, a mixed system of size $n$ can arise by mixing arbitrary coherent systems of this size. The family of proper 'deterministic' 
coherent systems is a finite subset of the set of all mixed systems. So we consider below arbitrary mixed systems with lifetime distribution function (1) and arbitrary signature (2). Given $s=\left(s_{1}, \ldots, s_{n}\right)$, we define distribution function (5) and its density function (6). Note that the former is a polynomial of degree $n$ such that $G(0)=0$ and $G(1)=1$. Therefore, the auxiliary functions

$$
\begin{aligned}
& G_{1}(u)=\frac{G(u)}{u}=n \sum_{k=0}^{n-1}\left(\frac{1}{k+1} \sum_{i=1}^{k+1} s_{i}\right) B_{k, n-1}(u), \\
& G_{2}(u)=\frac{1-G(u)}{1-u}=n \sum_{k=0}^{n-1}\left(\frac{1}{n-k} \sum_{i=k+1}^{n} s_{i}\right) B_{k, n-1}(u)
\end{aligned}
$$

are polynomials of degree $n-1$, positive on $(0,1)$. Note that the right-hand sides of (7) and (8) allow us to define both the functions on the closed interval $[0,1]$. At the endpoints, we have

$$
\begin{aligned}
& G_{1}(0)=g(0)=n s_{1}, \quad G_{1}(1)=1, \\
& G_{2}(0)=1, \quad G_{2}(1)=g(1)=n s_{n} .
\end{aligned}
$$

With the above notation, we are in a position to formulate the main result of this paper.

Proposition 1. Let $T$ denote the lifetime of a mixed system with signature $\boldsymbol{s}=\left(s_{1}, \ldots, s_{n}\right)$, composed of $n$ elements with independent, identically continuously distributed lifetimes with a finite variance $\sigma^{2}>0$. Under the notation given in (7) and (8), the following inequality holds:

$$
\frac{\operatorname{var} T}{\sigma^{2}} \leq \max _{0 \leq u \leq v \leq 1} G_{1}(u) G_{2}(v)
$$

Proof. The idea of the inequality proof is taken from Papadatos (1995), who established analogous bounds for order statistics ( $k$-out-of- $n$ systems). We use the Hoeffding (1940) representations of lifetime variances of the single component,

$$
\operatorname{var} X_{1}=2 \iint_{\{x \leq y\}} F(x)[1-F(y)] \mathrm{d} x \mathrm{~d} y,
$$

and the mixed system,

$$
\operatorname{var} T=2 \iint_{\{x \leq y\}} G(F(x))[1-G(F(y))] \mathrm{d} x \mathrm{~d} y .
$$

Since $G$ attains the extreme values 0 and 1 only at 0 and 1 , respectively, we can write

$$
\begin{aligned}
\operatorname{var} T= & 2 \iint_{\{0<F(x), x \leq y, F(y)<1\}} G(F(x))[1-G(F(y))] \mathrm{d} x \mathrm{~d} y \\
\leq & \sup _{0<F(x) \leq F(y)<1} \frac{G(F(x))[1-G(F(y))]}{F(x)[1-F(y)]} \\
& \times 2 \iint_{\{0<F(x), x \leq y, F(y)<1\}} F(x)[1-(F(y))] \mathrm{d} x \mathrm{~d} y \\
= & \sup _{0<u \leq v<1} \frac{G(u)[1-G(v)]}{u(1-v)} 2 \iint_{\{x \leq y\}} F(x)[1-(F(y))] \mathrm{d} x \mathrm{~d} y \\
= & \max _{0 \leq u \leq v \leq 1} G_{1}(u) G_{2}(v) \sigma^{2} .
\end{aligned}
$$


Kochar et al. (1999) proved that if $T$ is the lifetime of a coherent system with signature $\boldsymbol{s}=\left(s_{1}, \ldots, s_{n}\right)$ then the signature of its dual system is $\boldsymbol{s}^{d}=\left(s_{n}, \ldots, s_{1}\right)$ (the formal definition of a dual system can be found in Barlow and Proschan (1975)). Similarly, we say that the dual system to the mixed system with signature $s=\left(s_{1}, \ldots, s_{n}\right)$ is the system with signature $s^{d}=$ $\left(s_{n}, \ldots, s_{1}\right)$. Consequently, the lifetime $T^{d}$ of the dual mixed system is equal to $X_{1: n}, \ldots, X_{n: n}$ with respective probabilities $s_{n}, \ldots, s_{1}$.

Corollary 1. Bound (9) is identical for mutually dual mixed systems.

Proof. By definition, the system dual to one with signature $s=\left(s_{1}, \ldots, s_{n}\right)$ and lifetime distribution function $G(F(x))$ has lifetime distribution function $G^{d}(F(x))=1-G(1-F(x))$. Hence,

$$
\begin{aligned}
\sup _{0<u \leq v<1} \frac{G^{d}(u)\left[1-G^{d}(v)\right]}{u(1-v)} & =\sup _{0<u \leq v<1} \frac{[1-G(1-u)] G(1-v)}{u(1-v)} \\
& =\sup _{0<s \leq t<1} \frac{[1-G(t)] G(s)}{(1-t) s},
\end{aligned}
$$

and the statement follows.

Below we present an efficient numerical method of calculating bound (9) of Proposition 1. Let $0<a_{1}<\cdots<a_{k}<1$ and $0<b_{1}<\cdots<b_{l}<1$ be all the points at which the derivatives

$$
\begin{aligned}
& G_{1}^{\prime}(u)=n(n-1) \sum_{k=0}^{n-2} \frac{1}{k+2}\left(s_{k+2}-\frac{1}{k+1} \sum_{i=1}^{k+1} s_{i}\right) B_{k, n-2}(u), \\
& G_{2}^{\prime}(u)=n(n-1) \sum_{k=0}^{n-2} \frac{1}{n-k}\left(\frac{1}{n-k-1} \sum_{i=k+2}^{n} s_{i}-s_{k+1}\right) B_{k, n-2}(u)
\end{aligned}
$$

of (7) and (8), respectively, change their signs. Let $0<x_{1}<\cdots<x_{m}<1$ arise by combining all the elements of sequences $a_{i}, i=1, \ldots, k$, and $b_{j}, j=1, \ldots, l$. Note that $0 \leq m \leq 2 n-4$, and set $x_{0}=0$ and $x_{m+1}=1$. Each $\left[x_{i-1}, x_{i}\right], i=1, \ldots, m+1$, is the maximal interval where both (7) and (8) are monotone. Let $V_{p}=\left(x_{i_{p}}, x_{j_{p}}\right), p=1, \ldots, q$, be all the pairs such that $x_{i_{p}}<x_{j_{p}}$, and (7) and (8) have local maxima at $x_{i_{p}}$ and $x_{j_{p}}$, respectively. In other words, both the derivatives in (12) and (13) change their signs from plus to minus at $x_{i_{p}}$ and $x_{j_{p}}$, respectively. Let $I_{r}=\left[x_{i_{r}-1}, x_{i_{r}}\right], r=1, \ldots, s$, be the intervals where (12) is nonnegative and (13) is nonpositive. Then we have the following proposition.

Proposition 2. Under the above notation,

$$
\begin{aligned}
\max _{0 \leq u \leq v \leq 1} G_{1}(u) G_{2}(v)=\max \left\{G_{1}\left(x_{i_{p}}\right) G_{2}\left(x_{j_{p}}\right), p\right. & =1, \ldots, q, \\
& \left.\max _{x_{i_{r}-1 \leq u \leq x_{i r}}} G_{1}(u) G_{2}(u), r=1, \ldots, s\right\} .
\end{aligned}
$$

The proposition asserts that in order to establish the bound in Proposition 1, it suffices to determine all the pairs of local maxima of the factors $G_{1}$ and $G_{2}$ with properly ordered arguments, and compare the products with the extremal value of the product over the diagonal, which is a polynomial of degree $2 n-2$ of a single variable there. Moreover, it shows to which parts of the diagonal the search for the maximum can be restricted. 
Proof of Proposition 2. The proof is based on elementary arguments. We partition the triangle $\Delta=\{(u, v): 0 \leq u \leq v \leq 1\}$ into the families of rectangles

$$
R_{i, j}=\left\{(u, v): x_{i-1} \leq u \leq x_{i}, x_{j-1} \leq v \leq x_{j}\right\}, \quad 1 \leq i<j-1 \leq m,
$$

and triangles

$$
\Delta_{i}=\left\{(u, v): x_{i-1} \leq u \leq v \leq x_{i}\right\}, \quad 1 \leq i \leq m+1,
$$

with disjoint interiors and possibly common edges. It is easy to see that the maximum of $G_{1}(u) G_{2}(v)$ over an arbitrary rectangle $R_{i, j}$ is attained at either of its vertices. For instance, if $G_{1}$ and $G_{2}$ are increasing in $\left[x_{i-1}, x_{i}\right]$ and $\left[x_{j-1}, x_{j}\right]$, respectively, then we increase the product by increasing either of the variables until we reach the maximum at the right upper vertex. Starting from a point of triangle $\Delta_{i}$, and performing the naive maximization procedure, we can stop either at the right upper vertex (if $G_{1}$ and $G_{2}$ are increasing in $\left[x_{i-1}, x_{i}\right]$ ) or at the left lower vertex (if $G_{1}$ and $G_{2}$ are decreasing) or at the left upper vertex (if $G_{1}$ is decreasing and $G_{2}$ is increasing). Lastly, we stop on the hypotenuse edge if $G_{1}$ is increasing and $G_{2}$ is decreasing. When we arrive at a vertex of a rectangle or triangle, we can treat it as a border point of a neighboring figure, and repeat the procedure. It is easy to note that we stop at a nondiagonal point of the whole domain $\Delta$ if it is the common vertex of either four (if it is an interior point of $\Delta$ ) or two (if it is a point of either of legs of $\Delta$ ) rectangles, and it maximizes the product over all these rectangles. This clearly gives a local maximum of both the factors. The maximization over the diagonal may be restricted to the intervals, where (7) is increasing and (8) is decreasing.

Below we discuss attainability of the bound in Proposition 1.

Proposition 3. Bound (9) is sharp if and only if the maximum of the right-hand side is attained at a point of the diagonal line segment $D=\{(u, u): 0 \leq u \leq 1\}$.

This proposition means that for the systems for which the maximum is attained apart from the diagonal, the general maximum can be treated as an upper estimate of the sharp bound, whereas the maximum constrained to the diagonal may serve as a lower estimate of the unknown sharp upper bound.

Proof of Proposition 3. Suppose first that

$$
\max _{0 \leq u \leq v \leq 1} G_{1}(u) G_{2}(v)=G_{1}\left(u_{0}\right) G_{2}\left(u_{0}\right)
$$

for some $0<u_{0}<1$. Repeating the arguments of Papadatos (1995), we note that the equality in (11) holds if and only if either $F(x)[1-F(y)]=0$ or $F(x)[1-F(y)]=u_{0}\left(1-u_{0}\right)$ almost everywhere with respect to the Lebesgue measure on the half-space $\{(x, y): x \leq y\}$. This implies that $F$ can take on only three values, $0, u_{0}$, and 1 . So $F$ determines two-point distributions with the probability of the smaller point equal to $u_{0}$. Let $F_{0}$ be such a exemplary distribution function with the respective quantile function

$$
F_{0}^{-1}(u)= \begin{cases}a, & 0<u<u_{0}, \\ b, & u_{0} \leq u<1,\end{cases}
$$

for some $0<a<b$. Let $F_{k}, k=1,2, \ldots$, be a sequence of continuous distribution functions such that their quantile functions tend to $F_{0}^{-1}$ in the norm of $L^{2}((0,1), \mathrm{d} x)$. For instance, $F_{k}$ 
can be chosen as the mixture of uniform distributions over the intervals $[a-1 / k, a+1 / k]$ and $[b-1 / k, b+1 / k]$ with respective probabilities $u_{0}$ and $1-u_{0}$. Then we have

$$
\begin{gathered}
\mathrm{E}_{k} X_{1}=\int_{0}^{1} F_{k}^{-1}(u) \mathrm{d} u \rightarrow \int_{0}^{1} F_{0}^{-1}(u) \mathrm{d} u=a u_{0}+b\left(1-u_{0}\right), \\
\mathrm{E}_{k} X_{1}^{2}=\int_{0}^{1}\left[F_{k}^{-1}(u)\right]^{2} \mathrm{~d} u \rightarrow \int_{0}^{1}\left[F_{0}^{-1}(u)\right]^{2} \mathrm{~d} u=a^{2} u_{0}+b^{2}\left(1-u_{0}\right),
\end{gathered}
$$

and

$$
\operatorname{var}_{k} X_{1} \rightarrow u_{0}\left(1-u_{0}\right)(b-a)^{2}
$$

Similarly,

$$
\begin{gathered}
\mathrm{E}_{k} T=\int_{0}^{1} F_{k}^{-1}(u) g(u) \mathrm{d} u \rightarrow a G\left(u_{0}\right)+b\left[1-G\left(u_{0}\right)\right], \\
\mathrm{E}_{k} T^{2}=\int_{0}^{1}\left[F_{k}^{-1}(u)\right]^{2} g(u) \mathrm{d} u \rightarrow a^{2} G\left(u_{0}\right)+b^{2}\left[1-G\left(u_{0}\right)\right], \\
\operatorname{var}_{k} T \rightarrow G\left(u_{0}\right)\left[1-G\left(u_{0}\right)\right](b-a)^{2},
\end{gathered}
$$

and, consequently,

$$
\frac{\operatorname{var}_{k} T}{\operatorname{var}_{k} X_{1}} \rightarrow \frac{G\left(u_{0}\right)\left[1-G\left(u_{0}\right)\right]}{u_{0}\left(1-u_{0}\right)}=G_{1}\left(u_{0}\right) G_{2}\left(u_{0}\right) .
$$

Suppose now that

$$
\max _{0 \leq u \leq v \leq 1} G_{1}(u) G_{2}(v)=G_{1}(0) G_{2}(0)=\lim _{u \searrow 0} \frac{G(u)[1-G(u)]}{u(1-u)} .
$$

For fixed $0<a<b$ and $u_{l} \searrow 0$, we consider

$$
F_{l}^{-1}(u)=\left\{\begin{array}{ll}
a, & 0<u<u_{l}, \\
b, & u_{l} \leq u<1,
\end{array} \quad l=1,2 \ldots,\right.
$$

and, for each $F_{l}^{-1}$, we construct a sequence of continuous distributions with quantile functions $F_{l, k}^{-1}, k=1,2, \ldots$, approaching $F_{l}^{-1}$ in the sense described in the previous paragraph. We can easily check that

$$
\lim _{k \rightarrow \infty} \frac{\operatorname{var}_{k, k} T}{\operatorname{var}_{k, k} X_{1}}=\lim _{k \rightarrow \infty} \frac{G\left(u_{k}\right)\left[1-G\left(u_{k}\right)\right]}{u_{k}\left(1-u_{k}\right)}=G_{1}(0) G_{2}(0) .
$$

A similar reasoning applies to the case when the maximum is attained at $(1,1)$.

Now we show that bound (9) is not sharp if the respective maximum is attained in the interior of the triangle. Suppose that

$$
\begin{aligned}
m: & =\max _{0 \leq u \leq 1} G_{1}(u) G_{2}(u) \\
& =\sup _{0<u<1} \frac{G(u)[1-G(u)]}{u(1-u)} \\
& <M:=\max _{0<u<v<1} G_{1}(u) G_{2}(v) \\
& =\max _{0<u<v<1} \frac{G(u)[1-G(v)]}{u(1-v)} .
\end{aligned}
$$


The latter maximum is attained at a finite number of pairs $0<u_{p}<v_{p}<1, p=1, \ldots, q$, bounded by the total number of pairs of local extrema of polynomials $G_{1}$ and $G_{2}$. We prove that there does not exist a distribution function $F$ (possibly improper, with an atom at $\infty$ ) for which the equality in (11) holds. This would imply that the equality is not attainable in the limit by any sequence of continuous distribution functions.

The equality in (11) holds if and only if

$$
\frac{G(F(x))[1-G(F(y))]}{F(x)(1-F(y))}=M
$$

almost everywhere with respect to the Lebesgue measure on the set $\{0<F(x), x \leq y$, $F(y)<1$. This has a positive measure, because (10) is positive. Relation (14) means that either $F(x)=u_{p}$ or $F(y)=v_{p}, p=1, \ldots, q$, holds almost everywhere, and, consequently, at least one of the sets $\left\{(x, y): F(x)=u_{p}, F(y)=v_{p}\right\}$ for some $p$ has a positive measure. By monotonicity of $F$, it contains a rectangle, $\left[x_{1}, x_{2}\right] \times\left[y_{1}, y_{2}\right]$ say, of positive measure. Hence, $F(x)=u_{p}, x_{1} \leq x \leq x_{2}$, and $F(x)=v_{p}, y_{1} \leq x \leq y_{2}$. Furthermore, relations

$$
\begin{aligned}
& \frac{G(F(x))[1-G(F(y))]}{F(x)[1-F(y)]}=\frac{\left.G\left(u_{p}\right)\left[1-G\left(u_{p}\right)\right)\right]}{u_{p}\left(1-u_{p}\right)} \leq m<M, \\
& \frac{G(F(x))[1-G(F(y))]}{F(x)[1-F(y)]}=\frac{\left.G\left(v_{p}\right)\left[1-G\left(v_{p}\right)\right)\right]}{v_{p}\left(1-v_{p}\right)} \leq m<M
\end{aligned}
$$

hold on triangles $x_{1} \leq x \leq y \leq x_{2}$ and $y_{1} \leq x \leq y \leq y_{2}$ of positive measures $\left(x_{2}-x_{1}\right)^{2} / 2$ and $\left(y_{2}-y_{1}\right)^{2} / 2$, respectively. This contradicts the fact that (14) holds almost everywhere.

More subtle analysis should be carried out if the maximum of (9) is attained only on the line $\{(0, v): 0<v \leq 1\}$ or the line $\{(u, 1): 0 \leq u<1\}$. If, for example, $\left(0, v_{0}\right)$ is a minimum point then

$$
\begin{aligned}
\sup _{0<u<v<\min \{u+\delta, 1\}} \frac{G(u)[1-G(u)]}{u(1-u)} & \leq m+\varepsilon \\
& <M-\varepsilon \\
& \leq \sup _{0<u<\delta} \frac{G(u)\left[1-G\left(v_{0}\right)\right]}{u\left(1-v_{0}\right)}
\end{aligned}
$$

for some positive $\delta$ and $\varepsilon$. A detailed argumentation is left to the reader.

Finally we mention two extensions of our main result given in Proposition 1. Firstly, we observe that Proposition 1 can also be applied to systems with i.i.d. component lifetimes even if the common distribution is not continuous. This is based on the fact that relations (1) and (4) hold in this case when we use the signature vector $s=\left(s_{1}, \ldots, s_{n}\right)$ computed in the case of independent and identically continuously distributed component lifetimes (see Navarro et al. (2008b)). Secondly, we note that Proposition 1 can be applied for determining bounds on the residual variance of a mixed system $\operatorname{var}(T-t \mid T>t)$ by use of a representation of the residual lifetime $(T-t \mid T>t)$ distribution due to Navarro et al. (2008a, Theorem 2.5).

\section{Special cases}

We first recall the variation diminishing property of Bernstein polynomials, proved in Schoenberg (1959). The following form convenient for our purposes can be found in Rychlik (2001, p. 66). 
Lemma 1. The number of zeros of a given nonzero linear combination of Bernstein polynomials,

$$
B(u)=\sum_{k=0}^{m} a_{k} B_{k, m}(u), \quad 0<u<1,
$$

does not exceed the number of sign changes of the sequence $a_{0}, \ldots, a_{m}$. The first and last signs of (15) are the same as the signs of the first and last nonzero elements of $a_{0}, \ldots, a_{m}$, respectively.

The variance evaluations for special cases are based on analyzing properties of distribution function (5) for specific $s=\left(s_{1}, \ldots, s_{n}\right)$. Its first and second derivatives are (6) and

$$
g^{\prime}(u)=n(n-1) \sum_{k=0}^{n-2}\left(s_{k+2}-s_{k+1}\right) B_{k, n-2}(u) .
$$

Application of Lemma 1 to (16) simplifies the analysis. The corollaries below show that, for many systems, a cursory look on the system signature provides an immediate solution of the variance bound problem, (9).

Corollary 2. If (6) is decreasing (in particular, when $s=\left(s_{1}, \ldots, s_{n}\right)$ is nonincreasing) then the inequality $\operatorname{var} T / \sigma^{2} \leq n s_{1}$ is sharp.

Proof. First note that a nonincrease of $s$, combined with Lemma 1 and (16), implies a decrease of (6). Assume now that (6) is decreasing and that (5) is strictly concave. Take any $0<u_{1}<u_{2}<1$. The point $\left(u_{1}, G\left(u_{1}\right)\right)$ lies above the line segment connecting $(0, G(0))=$ $(0,0)$ with $\left(u_{2}, G\left(u_{2}\right)\right)$, and so does the segment joining $(0,0)$ with $\left(u_{1}, G\left(u_{1}\right)\right)$. So we have the following relation for the slopes:

$$
\frac{G\left(u_{1}\right)}{u_{1}}>\frac{G\left(u_{2}\right)}{u_{2}} .
$$

By similar arguments we check that

$$
\frac{1-G\left(u_{1}\right)}{1-u_{1}}>\frac{1-G\left(u_{2}\right)}{1-u_{2}} .
$$

This implies that both $G_{1}(u)=G(u) / u$ and $G_{2}(u)=[1-G(u)] /(1-u)$ are decreasing on $(0,1)$, and so

$$
\max _{0 \leq u \leq v \leq 1} G_{1}(u) G_{2}(v)=G_{1}(0) G_{2}(0)=n s_{1} .
$$

A construction of a sequence of distributions attaining the bound in the limit was presented in the proof of Proposition 3.

Analogously, we prove the following corollary.

Corollary 3. If (6) is increasing (in particular, when $s=\left(s_{1}, \ldots, s_{n}\right)$ is nondecreasing) then the inequality $\operatorname{var} T / \sigma^{2} \leq n s_{n}$ is sharp.

Note that Corollaries 2 and 3 provide the following simple bounds on variances of series and parallel systems:

$$
\frac{\operatorname{var} X_{1: n}}{\sigma^{2}} \leq n, \quad \frac{\operatorname{var} X_{n: n}}{\sigma^{2}} \leq n
$$

(cf. Papadatos (1995)). Respective evaluations for other $k$-out-of- $n$ systems follow from the next corollary. 
Corollary 4. Assume that (6) is first increasing and then decreasing.

(i) If $n s_{1}<1$ and $n s_{n}<1$, then there exist $u_{1}$ and $u_{2}, 0<u_{1}<u_{2}<1$, determined uniquely by the equations

$$
\begin{aligned}
& G_{2}(u)=g(u), \\
& G_{1}(u)=g(u),
\end{aligned}
$$

respectively, and $u_{1} \leq u_{0} \leq u_{2}$ such that the bound

$$
\frac{\operatorname{var} T}{\sigma^{2}} \leq G_{1}\left(u_{0}\right) G_{2}\left(u_{0}\right)
$$

is sharp.

(ii) If $n s_{n}<1 \leq n s_{1}$ then there exists a unique solution $0<u_{2}<1$ to (18), and $0 \leq u_{0} \leq u_{2}$ such that inequality (19) is sharp.

(iii) If $n s_{1}<1 \leq n s_{n}$ then there exists a unique solution $0<u_{1}<1$ to (17) such that, for some $u_{1} \leq u_{0} \leq 1$, inequality (19) is sharp.

Observe that the assumption that the signature sequence is first nondecreasing and then nonincreasing implies that (6) is increasing-decreasing. Note that it also implies either conditions (i)-(iii) or $s_{1}=\cdots=s_{n}=1 / n$, which leads to the trivial identity $\operatorname{var} T=\operatorname{var} X_{1}$.

Proof of Corollary 4. By assumption, (5) is strictly increasing, first convex and then concave. Under (i), its graph lies below and above the diagonal of the unit cube $[0,1]^{2}$ at the right and left neighborhoods of 0 and 1 , respectively. The curve crosses the diagonal at a single point. A thorough, but elementary, analysis of the graph leads to the conclusion that the slopes $G_{1}(u)=G(u) / u$ of the lines joining $(0,0)$ with the graph points $(u, G(u)), 0<u \leq 1$, first increase from $n s_{1}$ over the whole convexity domain and continue to increase to a maximum greater than 1 , and ultimately decrease to 1 at 1 . The maximum is attained at the unique point at which the line is tangent to the curve, and this is determined by (18). Similarly, we check that $G_{2}(u)=(1-G(u)) /(1-u)$ first increases from 1 at 0 to a maximal value $\left(1-G\left(u_{1}\right)\right) /\left(1-u_{1}\right)=g\left(u_{1}\right)>1$, and then decreases to $n s_{n}$. Point $u_{1}$ precedes the inflection point of $G$. By Proposition 2 we partition $[0,1]$ into three intervals, $\left[0, u_{1}\right],\left[u_{1}, u_{2}\right]$, and $\left[u_{2}, 1\right]$. Both $G_{1}$ and $G_{2}$ are increasing on $\left[0, u_{1}\right]$, and both are decreasing on $\left[u_{2}, 1\right]$. In the middle interval, $G_{1}$ increases and $G_{2}$ decreases. The unique pair of their local extrema $\left(u_{2}, u_{1}\right)$ lies beyond the triangle domain of maximization. The statement of Proposition 2 asserts that the constrained maximum of the right-hand side of (9) is located on the central part $\left\{(u, u): u_{1} \leq u \leq u_{2}\right\}$ of the diagonal. By Proposition 3, the respective bound is sharp.

In case (ii) the whole graph of the convex-concave function $G$ lies above the diagonal. As before, $G_{1}$ first increases on $\left[0, u_{2}\right]$ and eventually decreases, but $G_{2}$ is now decreasing on the whole interval. By Proposition 2, the maximum point $\left(u_{0}, u_{0}\right)$ is located on the lower part $\left\{(u, u): 0 \leq u \leq u_{2}\right\}$ of the diagonal, and the respective bound is sharp. In the last case, the curve runs below the line, $G_{1}$ is increasing, and $G_{2}$ is increasing and decreasing on $\left[0, u_{1}\right]$ and $\left[u_{1}, 1\right]$, respectively. By analogous arguments, the last conclusion follows.

The sharp bounds for $k$-out-of- $n$ systems, $2 \leq k \leq n-1$, can be deduced from Corollary 4(i). In fact, Papadatos (1995) managed to prove that in these cases $G_{1}(u) G_{2}(u), u_{1} \leq u \leq u_{2}$, is maximized by a unique point at which the derivative of the product vanishes. We are not able to prove an analogous claim in the case of general unimodal signature vectors. 
A majority of systems have signatures satisfying the assumptions of the corollaries. All the coherent systems of sizes up to four have either monotone or unimodal signature vectors. Consequently, the respective density functions (6) are either monotone or unimodal, and the bounds of Proposition 1 are sharp for these systems. The numerical values of the bounds are presented in Table 1. In each row, for a given system, represented by the functional dependence of its lifetime $T$ on $X_{1}, \ldots, X_{n}$, we present the consecutive values of corresponding signature coefficients, the variance bound, and the parameter $u_{0}$ describing the limiting twopoint distribution attaining the bound. This parameter represents the probability of the smaller of two points. Values $u_{0}=0$ or 1 respectively mean that the probabilities of the smaller value should tend to 0 or 1 in order to reach the bound in the limit.

TABLE 1: Bounds on variances of coherent systems with $n=2,3,4$ components.

\begin{tabular}{|c|c|c|c|c|c|c|}
\hline System & $s_{1}$ & $s_{2}$ & $s_{3}$ & $s_{4}$ & Bound & $u_{0}$ \\
\hline$X_{1: 2}$ & 1 & 0 & & & 2 & 0 \\
\hline$X_{2: 2}$ & 0 & 1 & & & 2 & 1 \\
\hline$X_{1: 3}$ & 1 & 0 & 0 & & 3 & 0 \\
\hline $\min \left(X_{1}, \max \left(X_{2}, X_{3}\right)\right)$ & $\frac{1}{3}$ & $\frac{2}{3}$ & 0 & & 1.11493 & 0.22367 \\
\hline$X_{2: 3}$ & 0 & 1 & 0 & & 1 & 0.5 \\
\hline $\max \left(X_{1}, \min \left(X_{2}, X_{3}\right)\right)$ & 0 & $\frac{2}{3}$ & $\frac{1}{3}$ & & 1.11493 & 0.77633 \\
\hline$X_{3: 3}$ & 0 & 0 & 1 & & 3 & 1 \\
\hline$X_{1: 4}$ & 1 & 0 & 0 & 0 & 4 & 0 \\
\hline $\min \left(X_{1}, X_{2}, \max \left(X_{3}, X_{4}\right)\right)$ & $\frac{1}{2}$ & $\frac{1}{2}$ & 0 & 0 & 2 & 0 \\
\hline $\min \left(X_{1}, \max _{2 \leq i<j \leq 4} \min \left(X_{i}, X_{j}\right)\right)$ & $\frac{1}{4}$ & $\frac{3}{4}$ & 0 & 0 & 1.27086 & 0.18298 \\
\hline $\min \left(X_{1}, \max \left(X_{2}, X_{3}\right), \max \left(X_{2}, X_{4}\right)\right)$ & $\frac{1}{4}$ & $\frac{7}{12}$ & $\frac{1}{6}$ & 0 & 1.19529 & 0.19763 \\
\hline $\min \left(X_{1}, \max \left(X_{2}, X_{3}, X_{4}\right)\right)$ & $\frac{1}{4}$ & $\frac{1}{4}$ & $\frac{1}{2}$ & 0 & 1.03459 & 0.31690 \\
\hline$X_{2: 4}$ & 0 & 1 & 0 & 0 & 1.08702 & 0.32530 \\
\hline $\begin{array}{c}\max \left(\min \left(X_{1}, X_{2}\right), \min \left(X_{1}, X_{3}, X_{4}\right)\right. \\
\left.\min \left(X_{2}, X_{3}, X_{4}\right)\right)\end{array}$ & 0 & $\frac{5}{6}$ & $\frac{1}{6}$ & 0 & 1.04370 & 0.36926 \\
\hline $\max \left(\min \left(X_{1}, X_{2}\right), \min \left(X_{3}, X_{4}\right)\right)$ & 0 & $\frac{2}{3}$ & $\frac{1}{3}$ & 0 & 1.01203 & 0.42798 \\
\hline $\begin{array}{c}\max \left(\min \left(X_{1}, X_{2}\right), \min \left(X_{1}, X_{3}\right)\right. \\
\left.\min \left(X_{2}, X_{3}, X_{4}\right)\right) \\
\max \left(\min \left(X_{1}, X_{2}\right), \min \left(X_{2}, X_{3}\right)\right.\end{array}$ & 0 & $\frac{2}{3}$ & $\frac{1}{3}$ & 0 & 1.01203 & 0.42798 \\
\hline $\begin{array}{c}\left.\min \left(X_{3}, X_{4}\right)\right) \\
\max \left(\min \left(X_{1}, \max \left(X_{2}, X_{3}, X_{4}\right)\right)\right.\end{array}$ & 0 & $\frac{1}{2}$ & $\frac{1}{2}$ & 0 & 1 & 0.5 \\
\hline $\begin{array}{c}\max \left(\min \left(X_{1}, \max \left(X_{2}, X_{3}, X_{4}\right)\right),\right. \\
\left.\min \left(X_{2}, X_{3}, X_{4}\right)\right) \\
\max \left(\min \left(X_{1}, \max \left(X_{2}, X_{3}, X_{4}\right)\right),\right.\end{array}$ & 0 & $\frac{1}{2}$ & $\frac{1}{2}$ & 0 & 1 & 0.5 \\
\hline $\left.\min \left(X_{2}, X_{3}\right)\right)$ & 0 & $\frac{1}{3}$ & $\frac{2}{3}$ & 0 & 1.01203 & 0.57202 \\
\hline $\begin{array}{c}\min \left(\max \left(X_{1}, X_{2}\right), \max \left(X_{3}, X_{4}\right)\right) \\
\min \left(\max \left(X_{1}, X_{2}\right), \max \left(X_{1}, X_{3}, X_{4}\right)\right.\end{array}$ & 0 & $\frac{1}{3}$ & $\frac{2}{3}$ & 0 & 1.01203 & 0.57202 \\
\hline $\left.\max \left(X_{2}, X_{3}, X_{4}\right)\right)$ & 0 & $\frac{1}{6}$ & $\frac{5}{6}$ & 0 & 1.04370 & 0.63074 \\
\hline$X_{3: 4}$ & 0 & 0 & 1 & 0 & 1.08702 & 0.67470 \\
\hline $\max \left(X_{1}, \min \left(X_{2}, X_{3}, X_{4}\right)\right)$ & 0 & $\frac{1}{2}$ & $\frac{1}{4}$ & $\frac{1}{4}$ & 1.03459 & 0.68310 \\
\hline $\max \left(X_{1}, \min \left(X_{2}, X_{4}\right), \min \left(X_{3}, X_{4}\right)\right)$ & 0 & $\frac{1}{6}$ & $\frac{7}{12}$ & $\frac{1}{4}$ & 1.19529 & 0.80237 \\
\hline $\max \left(X_{1}, \max _{2 \leq i<j \leq 4} \min \left(X_{i}, X_{j}\right)\right)$ & 0 & 0 & $\frac{3}{4}$ & $\frac{1}{4}$ & 1.27086 & 0.81702 \\
\hline $\max \left(X_{1}, X_{2}, \min \left(X_{3}, X_{4}\right)\right)$ & 0 & 0 & $\frac{1}{2}$ & $\frac{1}{2}$ & 2 & 1 \\
\hline$X_{4: 4}$ & 0 & 0 & 0 & 1 & 4 & 1 \\
\hline
\end{tabular}


We have found examples of coherent systems with multimodal signatures (the simplest one is presented in Example 1, below), but all of them have unimodal densities (6) in the uniform case. This means that, for all of them, the bounds of Proposition 1 are sharp. We conjecture that for all 'deterministic' coherent systems, function (6) is either monotone or unimodal. On the other hand, there are 'random' mixed systems for which the variance bounds of Proposition 1 are not sharp. Two of them are presented in Examples 2 and 3, below. In Example 2, the maximum of the two-variable polynomial determining the bound is located at the border of the triangle, and in Example 3 it lies in its interior.

Example 1. Consider a system of five components with lifetime $T=\max \left(\min \left(X_{1}, \max \left(X_{2}\right.\right.\right.$, $\left.\left.\left.X_{3}, X_{4}, X_{5}\right)\right), \min \left(X_{2}, X_{3}, X_{4}, X_{5}\right)\right)$. The graph of the system is presented in Figure 1 . It has the signature vector $\left(0, \frac{2}{5}, \frac{1}{5}, \frac{2}{5}, 0\right)$.

The density

$$
g(u)=2 B_{1,4}(u)+B_{2,4}(u)+2 B_{3,4}(u)
$$

(cf. (6)) has derivative

$$
g^{\prime}(u)=8 B_{0,3}(u)-4 B_{1,3}(u)+4 B_{2,3}(u)-8 B_{3,3}(u)=4(1-2 u)\left[5\left(u-\frac{1}{2}\right)^{2}+\frac{3}{4}\right]
$$

(cf. (16)), which implies that the former is symmetric about $\frac{1}{2}$, and first increasing and eventually decreasing. Moreover,

$$
\begin{aligned}
G_{1}(u) & =B_{1,4}(u)+B_{2,4}(u)+\frac{5}{4} B_{3,4}(u)+B_{4,4}(u) \\
& =4 u-6 u^{2}+5 u^{3}-2 u^{4} \\
G_{2}(u) & =G_{1}(1-u) .
\end{aligned}
$$

The latter relation is a consequence of the fact that the signature vector is symmetric, i.e. $s_{n+1-i}=s_{i}, i=1, \ldots, n$, which implies that $G(1-u)=1-G(u)$. Applying Corollary 4 , we numerically determine the sharp variance bound

$$
\frac{\operatorname{var} T}{\sigma^{2}} \leq \max _{0 \leq u \leq 1} G_{1}(u) G_{2}(u)=G_{1}\left(\frac{1}{2}\right) G_{2}\left(\frac{1}{2}\right)=1
$$

attained in the limit when we approach a symmetric two-point distribution.

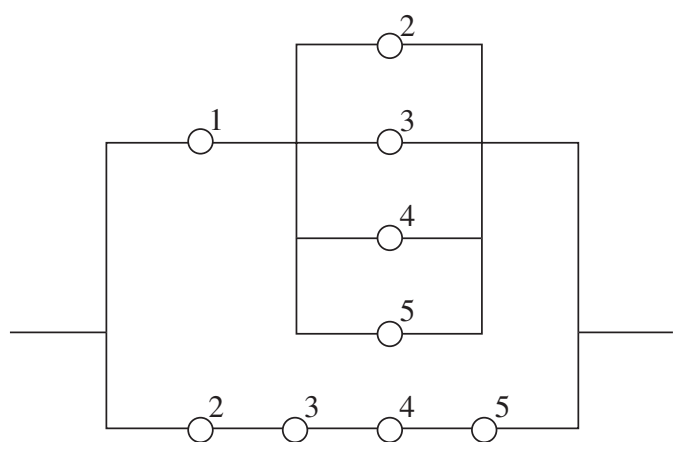

FIgURE 1: Coherent system of Example 1. 
Example 2. Take the mixed system which is a combination of series and parallel systems with signature defined by $s_{1}=s_{n}=\frac{1}{2}, s_{2}=\cdots=s_{n-1}=0$ for $n \geq 3$. Then

$$
\begin{aligned}
G(u) & =\frac{1}{2} \sum_{k=1}^{n-1} B_{k, n}(u)+B_{n, n}(u), \\
G_{1}(u) & =\sum_{k=0}^{n-2} \frac{n}{2(k+1)} B_{k, n-1}(u)+B_{n-1, n-1}(u), \\
G_{1}^{\prime}(u) & =-\sum_{k=0}^{n-3} \frac{n(n-1)}{2(k+1)(k+2)} B_{k, n-2}(u)+\frac{n-2}{2} B_{n-2, n-2}(u) .
\end{aligned}
$$

By Lemma 1, we deduce that $G_{1}^{\prime}(u)$ is first negative and then positive. Moreover,

$$
G_{1}^{\prime}\left(\frac{1}{2}\right)<\frac{n-2}{2} B_{n-2, n-2}\left(\frac{1}{2}\right)-\frac{n(n-1)}{4} B_{0, n-2}\left(\frac{1}{2}\right)=-\frac{(n-3 / 2)^{2}+7 / 4}{2^{n}}<0 .
$$

It follows that (20) decreases from $G_{1}(0)=n / 2$ to a positive minimum at some $\frac{1}{2}<u_{0}<1$, at which (20) vanishes, and then increases to $G_{1}(1)=1$. We have $G(1-u)=1-G(u)$ and $G_{2}(u)=G_{1}(1-u)$. This means that $G_{2}$ is also decreasing and increasing with two maxima $G_{2}(0)=1$ and $G_{2}(1)=n / 2$ at the ends, and one minimum at $0<1-u_{0}<\frac{1}{2}$. Applying Proposition 2, we conclude that the function $(u, v) \mapsto G_{1}(u) G_{2}(v), 0 \leq u \leq v \leq 1$, has three local maxima: one $G_{1}(0) G_{2}(1)=n^{2} / 4$ at the right-angle vertex and two $G_{1}(0) G_{2}(0)=$ $G_{1}(1) G_{2}(1)=n / 2$ at the other two vertices. Therefore, the inequality

$$
\frac{\operatorname{var} T}{\sigma^{2}} \leq \frac{n^{2}}{4}
$$

of Proposition 1 is not sharp. However, the sharp bound is not less than $n / 2$, which is attained in the limit by sequences of continuous distributions concentrating about two points, with the probability mass of one concentration region decreasing to 0 .

Example 3. Consider the mixture of 2-out-of-6 and 5-out-of-6 systems with signature $\left(0, \frac{1}{2}, 0,0, \frac{1}{2}, 0\right)$. Here

$$
g(u)=3 B_{1,5}(u)+3 B_{4,5}(u)
$$

is symmetric about $\frac{1}{2}$ and bimodal. It has two maxima at 0.21133 and 0.78867 , and a minimum at 0.5 . The function

$$
G_{1}(u)=\frac{3}{2} B_{1,5}(u)+B_{2,5}(u)+\frac{3}{4} B_{3,5}(u)+\frac{6}{5} B_{4,5}(u)+B_{5,5}(u)
$$

first increases from 0 at 0 to $G_{1}\left(u_{1}\right)=1.00960$ at $u_{1}=0.39879$, then decreases to $G_{1}\left(u_{2}\right)=$ 0.99346 at $u_{2}=0.58848$, and increases to $G_{1}\left(u_{3}\right)=1.04801$ at $u_{3}=0.88772$, and ultimately decreases to $G_{1}(1)=1$. Behavior of $G_{2}$ can be easily deduced from the relation $G_{2}(u)=G_{1}(1-u)$. Accordingly, there is a local maximum of $G_{1}(u) G_{2}(v)$ at the point $\left(u_{1}, 1-u_{1}\right)$ of the open triangle $0<u<v<1$, and this amounts to

$$
G_{1}\left(u_{1}\right) G_{2}\left(1-u_{1}\right)=G_{1}^{2}\left(u_{1}\right)=1.019297 .
$$

The product restricted to the diagonal $G_{1}(u) G_{2}(u), 0 \leq u \leq 1$, is symmetric about $\frac{1}{2}$ and bimodal. The maximal diagonal value

$$
G_{1}(0.38168) G_{2}(0.38168)=G_{1}(0.61832) G_{2}(0.61832)=1.00342
$$


is less. Therefore, the bound

$$
\frac{\operatorname{var} T}{\sigma^{2}} \leq 1.019297
$$

following from Proposition 1, is not sharp here.

The value 1.00342 is the best bound for two-point distributions. We can get a greater value when we consider, for instance, symmetric three-point distributions

$$
\mathrm{P}_{u}\left(X_{1}=a \pm b\right)=u, \quad \mathrm{P}_{u}\left(X_{1}=a\right)=1-2 u, \quad 0<u<\frac{1}{2} .
$$

We easily verify that

$$
\operatorname{var}_{u} X_{1}=2 u b^{2}, \quad \operatorname{var}_{u} T=\left[G_{2: 6}(u)+G_{5: 6}(u)\right] b^{2},
$$

and

$$
\sup _{0<u<1 / 2} \frac{\operatorname{var}_{u} T}{\operatorname{var}_{u} X_{1}}=\max _{0 \leq u \leq 1 / 2} G_{1}(u)=1.009602 \in(1.00342,1.019297),
$$

attained at $u_{1}=0.39879$.

Note that, taking into account limiting symmetric three-point distributions, we do not improve the lower approximation $n / 2$ of the sharp upper bound for the mixture of series and parallel systems of Example 2. The value $n^{2} / 4$ seems to be the greatest possible upper evaluation for variances of lifetimes of arbitrary mixed systems with $n$ components that follows from Proposition 1. It is significantly greater than $n$, which is the maximal upper estimate of Papadatos (1995) for all $k$-out-of- $n$ systems. It remains an open question if there exists a coherent or mixed system and a distribution function of component lifetimes such that $\operatorname{var} T / \sigma^{2}>n$.

On the other hand, for each system with an arbitrary signature, there are component lifetime distributions such that $\operatorname{var} T / \sigma^{2}>1-\varepsilon$ for arbitrarily small $\varepsilon>0$. To check the claim, it is sufficient to show that

$$
\max _{0 \leq u \leq 1} G_{1}(u) G_{2}(u) \geq 1
$$

by analyzing the cases where function (5) runs above, below, and crosses the straight line joining $(0,0)$ and $(1,1)$. In the first case, we have $G_{1}(0)=n s_{1} \geq 1$, and so $G_{1}(0) G_{2}(0)=n s_{1} \geq 1$. In the second case, $G_{1}(1) G_{2}(1)=n s_{n} \geq 1$. In the third case, for any crossing point $0<u_{0}<1$, we obtain $G_{1}\left(u_{0}\right) G_{2}\left(u_{0}\right)=1$.

\section{Acknowledgements}

The authors thank an anonymous referee for suggestions that allowed us to correct some mistakes and improve the presentation of the paper. The second author was partially supported by Ministerio de Educación y Ciencia under grant MTM2006-12834 and Fundación Séneca under grant 08627/PI/08. The third author was supported by the Polish Ministry of Science and Higher Education, grant number 1 P03A 01530.

\section{References}

Barlow, R. E. And Proschan, F. (1975). Statistical Theory of Reliability and Life Testing. Holt, Rinehart and Winston, New York.

Beare, B. K. (2009). A generalization of Hoeffding's lemma, and a new class of covariance inequalities. Statist. Prob. Lett. 79, 637-642.

Block, H. W. and Fang, Z. B. (1988). A multivariate extension of Hoeffding's lemma. Ann. Prob. 16, $1803-1820$. 
Boland, P. J. And Samaniego, F. J. (2004). The signature of a coherent system and its applications in reliability. In Mathematical Reliability: An Expository Perspective (Internat. Ser. Operat. Res. Manag. Sci. 67), eds R. Soyer, T. Mazzuchi and N. Singpurwalla, Kluwer, Boston, MA, pp. 3-30.

Cuadras, C. M. (2002). On the covariance between functions. J. Multivariate Anal. 81, 19-27.

Hoeffoing, W. (1940). Masstabinvariante korrelations-theorie. Schriften Math. Inst. Univ. Berlin 5, 181-233.

Jones, M. C. AND Balakrishnan, N. (2002). How are moments and moments of spacings related to distribution functions? J. Statist. Planning Infer. 103, 377-390.

KlimczaK, M. And Rychlik, T. (2004). Maximum variance of $k$ th records. Statist. Prob. Lett. 69, 421-430.

Kochar, S., Mukerjee, H. and Samaniego, F. J. (1999). The 'signature' of a coherent system and its application to comparisons among systems. Naval Res. Logistics 46, 507-523.

Lehmann, E. L. (1966). Some concepts of dependence. Ann. Math. Statist. 37, 1137-1153.

Mardia, K. V. And Thompson, J. W. (1972). Unified treatment of moment-formulae. Sankhyā 34, 121-132.

Navarro, J. AND Rubio, R. (2009). Computations of signatures of coherent systems with five components. To appear in Commun. Statist. Simul. Comput.

Navarro, J. AND Rychlik, T. (2007). Reliability and expectation bounds for coherent systems with exchangeable components. J. Multivariate Anal. 98, 102-113.

Navarro, J., Balakrishnan, N. And Samaniego, F. J. (2008a). Mixture representations of residual lifetimes of used systems. J. Appl. Prob. 45, 1097-1112.

Navarro, J., Ruiz, J. M. and Sandoval, C. J. (2007). Properties of coherent systems with dependent components. Commun. Statist. Theory Meth. 36, 175-191.

Navarro, J., Samaniego, F. J., Balakrishnan, N. and Bhattacharya, D. (2008b). On the application and extension of system signatures to problems in engineering reliability. Naval Res. Logistics 55, 313-327.

Papadatos, N. (1995). Maximum variance of order statistics. Ann. Inst. Statist. Math. 47, 185-193.

Papadatos, N. (1997). A note on maximum variance of order statistics from symmetric populations. Ann. Inst. Statist. Math. 49, 117-121.

Prakasa RaO, B. L. S. (1998). Hoeffding identity, multivariance and multicorrelation. Statistics 32, 13-29.

Quesada-Molina, J. J. (1992). A generalization of an identity of Hoeffding and some applications. J. Italian Statist. Soc. 3, 405-411.

Rychlik, T. (2001). Projecting Statistical Functionals (Lecture Notes Statist. 160). Springer, New York.

Rychlik, T. (2008a). Bounds on lifetimes of coherent systems with exchangeable components. In Advances in Mathematical Modeling for Reliability, eds T. Bedford et al., IOS Press, Amsterdam, pp. 111-118.

RyCHLIK, T. (2008b). Extreme variances of order statistics in dependent samples. Statist. Prob. Lett. 78, $1577-1582$.

Samaniego, F. (1985). On closure of the IFR class under formation of coherent systems. IEEE Trans. Reliab. R-34, 69-72.

Samaniego, F. (2007). System Signatures and Their Applications in Engineering Reliability (Internat. Ser. Operat. Res. Manag. Sci. 110). Springer, New York.

Schoenberg, I. J. (1959). On variation diminishing approximation methods. In On Numerical Approximation (Proc. Symp., Madison, 1958), ed. R. E. Langer, University of Wisconsin Press, Madison, pp. 249-274.

ShaKed, M. AND SuAREZ-Llorens, A. (2003). On the comparison of reliability experiments based on the convolution order. J. Amer. Statist. Assoc. 98, 693-702.

Yu, H. (1993). A Glivenko-Cantelli lemma and weak convergence for empirical processes of associated sequences. Prob. Theory Relat. Fields 95, 357-370. 